\title{
Measurement, Analysis, and Comparison of the Parcel Shipping Shock and Drop Environment of the United States Postal Service with Commercial Carriers
}

\author{
J. Singh,1 S. P. Singh,2 G. Burgess,2 and K. Saha2 \\ 1Assistant Professor, Industrial Technology, Cal Poly State University, San \\ Luis Obispo, CA, \\ ${ }_{2}$ Professors and Research Assistant, respectively, School of Packaging, \\ Michigan State University, East Lansing, MI.
}

\begin{abstract}
The past decade has shown a great increase in the number of direct to consumer shipments of products and packages. As a result, parcel delivery companies like DHL, FedEx, UPS, and the USPS have strengthened their presence in air transport to deliver products faster and through larger distances. Using cargo planes, they route packages from various destinations to large airport hubs, where they sort millions of packages and ship them to their destinations. There is a continuous need to quantify what happens to these packages as they are handled both manually during collection and delivery and on large high-speed conveying and sortation equipment at hubs. The dynamic events during these moves can cause damage. The information collected in this study helps packaging engineers design protective packaging. This study measured and compared shock and drop events for these carriers during next-day and two-day shipping service between Michigan and California. Data are presented in terms of drop heights associated with the 90, 95, and 99th percentile of occurrence for DHL, FedEx, UPS, and USPS. Impact orientation is also discussed. The data from this study are beneficial to new test methods being developed for drop testing of single parcel shipments by ASTM.
\end{abstract}

\section{Introduction}

The United States Postal Service (USPS) is the largest postal organization in the world, accounting for over $40 \%$ of all mail handled, transported, and delivered in the world by volume. It is also one of the top four parcel carriers in the United States, and has been operating for more than two centuries, and through this period, has grown and changed [1]. The USPS initiated the first air delivery of mail from New York to Washington, DC in the early 1900s. Today the top four parcel carriers in the world, namely DHL International

GmbH (DHL), Federal Express (FedEx), United Parcel Service (UPS), and USPS, offer many of the same services, the main difference being the prices and perhaps the delivery timings for various destinations [1-4]. These companies service the entire United States and worldwide. While FedEx is the largest air delivery company (over 3 million parcels by air a day), UPS is the largest parcel handling company (13 million parcels by ground and air), and DHL serves the most international destinations, USPS continues to be the largest transportation and material handling organization handling and transporting hundreds of millions of mail pieces a day. With an increase in parcel shipping directly to the consumer from either manufacturing sites or retailers, it is necessary to understand the physical conditions that packages and their contents are exposed to by various delivery companies.

Packaged goods are shipped from one place to another using various means of transportation. There has been a continuous increase over the past two decades in measuring the dynamic events that occur to packages in different transportation methods. These data offer very useful information to design and test packages for potential hazards such as drops and impacts. This study focused on measuring a new segment of the mid-sized and lightweight single parcel environment for USPS that had not been previously measured. Various researchers have investigated the distribution environment segments for various transport modes to get a better understanding of the effects of physical hazards on packages. This information provides a basis for developing test methods to design economical protective packaging. The following researches provide input into the dynamic nature of the parcel shipping environment. 
Goff developed performance requirements that were necessary for parcel post packages in 1974 [5]. The study recommended performance-based test methods that could be used to reduce physical damage to parcel post packages. Singh andVoss and Singh and Cheema measured the dynamics of small parcel environment in the UPS ground-shipping environment [6] and the UPS and FedEx air-shipping environment [7]. The study tested packages of different sizes and weights that were instrumented with drop-height recorders and then shipped through UPS and FedEx. The study showed that the highest drop height measured was $1.06 \mathrm{~m}$ and that the size of the package had no significant effect on the drop heights associated with medium and larger-size packages. However, smallsize and lighter-weight packages did experience higher drop heights. This was attributed to the use of automated handling for the larger and heavier packages in the UPS sorting environment. The smaller and lighter packages are often placed on top in the delivery truck and are therefore subject to higher drops. Singh et al., in a study of packages weighing less than $2.5 \mathrm{~kg}$ and five different sizes shipped through second-day Air Express concluded that package size and weight had no effect on measured drop heights for packages classified as small and lightweight [8]. Also warning labels reading "Fragile-Handle with Care" had no significant effect on the handling of packages. Singh and Singh in another study analyzed the next-day and second-day parcel shipments made for lightweight and mid-sized packages through commercial carriers (DHL, FedEx, and UPS) [9]. Since the past two years the USPS has become a major player in shipping parcels. The largest number of packages shipped annually occurs between October and December, coinciding with the Thanksgiving and Christmas seasons. The USPS in 2005 initiated a seven-day week for accepting parcels for shipment. As a result, it is important to quantify and compare the levels packages are seen and observed by this carrier as compared to the commercial carriers. The USPS is a federally supervised organization and is operated and managed by federal employees.

Various previous studies have also measured the drop heights for larger and heavier packages. Singh et al. measured the environment within UPS for packages weighing up to $64 \mathrm{~kg}$ [10]. In addition, the effect of label position on drop orientation has also been studied in parcel shipments for large and heavy packages [11]. However, there are presently no data available on mid-sized and lightweight packages for USPS. This study draws a comparison between the dynamic hazards for the various parcel shipping environments discussed above and the two types of service. ASTM is currently developing a new test method to simulate the drop heights and impacts packages undergo during single parcel delivery. The data from this study are beneficial to establishing test severity levels.

This study had the following objectives:

- Characterize the dynamics of the Express (guaranteed next day) and Priority (two to three-day delivery) shipping environment for mid-sized $(0.38 \mathrm{~m}$ by $0.34 \mathrm{~m}$ by $0.34 \mathrm{~m}$ ) and lightweight packages (less than $7 \mathrm{~kg}$ ) shipped by USPS within the United States.

- Provide recommended test levels for drop testing packages of this size and weight for USPS as compared to those observed for DHL, FedEx, and UPS [9].

\section{Instrumentation}

In order to achieve the objectives of this study, data recorders were used to collect dynamic data that could be used to develop test methods to simulate the next-day and second-day shipping environment for mid-sized and lightweight packages. There are several types of instruments available to measure dynamic events that packages experience during shipping and handling. These range from single-drop counters that only record if the package is dropped above a preset height, to recorders that measure impacts along all three axes of the package. The electronic recorders monitor acceleration-time histories for all events. One such commercially available recorder is the "Environmental Data Recorder-3C" (EDR3C) manufactured by Instrumented Sensor Technology, Okemos, MI [12]. The EDR-3C is a portable, digital sensor/ recorder designed to measure shock, vibration, temperature, humidity, and pressure in packages during shipping. Based on previous studies, the pre-trigger and post-trigger times to be recorded were set at 50 and $250 \mathrm{~ms}$, respectively. The sampling rate was $1000 \mathrm{~Hz}$ and the trigger level was $2 \mathrm{G}$ for all three axes. The test duration was set at 60 days and the recorder was set to record with an 
overwrite limit of 900 events. The overwrite parameter ensures that the EDR-3C records the most severe events if the recorder experiences more than 900 events.

\section{Test Package Shipments}

Test packages were used to ship the EDR-3C recorders to measure the different events they experience in the next-day and second-day small parcel environment. The EDR-3C units were encased in a high density polyethylene foam cushion. The cushions were placed on all six sides so that the unit was at the geometric center of a two-piece, blow-molded, double-walled carrying case with four metal latches. This carrying case was then contained in a doublewall regular slotted container (RSC) corrugated board box. The entire process of preparing the EDR-3C unit for shipment is as shown in Fig. 1. All recorders were placed in the same orientation in all packages for every shipment. The corrugated shipping boxes were sealed using 51-mm wide general purpose plastic box sealing tape. All instrumented packages were 0.38 $\mathrm{m}$ by $0.34 \mathrm{~m}$ by $0.34 \mathrm{~m}$ in external dimensions and weighed $6.8 \mathrm{~kg}$. The packages were shipped from East Lansing, MI to San Luis Obispo, CA. The data and comparisons are based on 32 trips for all four carriers. This study was conducted in 2005 .

\section{Results}

Based on the data collected, drop height, impact orientation, and frequency of occurrence were tabulated for each package. Data for

USPS parcel shipments are presented in combination with DHL, FedEx, and UPS data from the previous study [5]. Tables 1-3 and Figs. 2 and 3 shows the results for packages shipped in the next-day and second-day shipping environments for DHL, FedEx, UPS, and USPS. All three commercial carriers offer similar services by air. The number of drops experienced by the packages in the Express (next-day) environment for USPS (156) was comparable with the average of those for DHL, FedEx, and UPS (143). The Priority (second-day) shipments in the USPS environment, however, experienced 2.3 times lesser drops as compared to the average of those experienced in DHL, FedEx, and UPS shipments. During the data analysis, drop heights below $76 \mathrm{~mm}$ were ignored since they typically produce very little damage to the contents [7]. Low level impacts are generally simulated in vibration tests. Table 1 shows the number of impacts measured above $76 \mathrm{~mm}$, the highest drop height recorded during any one-way trip, and the 90th, 95th, and 99th percentile drop heights for all five package sizes. The term "90\% occurrence" means that $90 \%$ of all recorded drop heights were below this level.

Packages shipped through the Express (next-day) option provided by USPS, for example, experienced 156 impacts equivalent to a drop of $76 \mathrm{~mm}$ or higher in eight one-way trips. This amounts to nearly 20 impacts above $76 \mathrm{~mm}$ per trip. The majority of these impacts are from automated handling and sorting equipment. Only about a third of these are expected to be actual drops. The largest drop height recorded of $1.87 \mathrm{~m}$ to packages shipped by Priority (second-day) mail through USPS was likely a rare event.

The drop heights reported in Table 2 are based on averaging the results of eight one-way trips (four round trips from Michigan to California) for all couriers. This format is often used as the basis for lab simulated drop tests where drop testing is done in sequentially reduced levels, similar to how they occur in an actual shipment [8]. Averaging the results of the one-way trips is done because the lab simulated tests are intended to represent a typical one-way trip. Table 2 shows these averaged drop heights for packages shipped in the next-day and second-day shipping environments for DHL, FedEx, UPS, and USPS. The data show that the highest drop height was greater for DHL and FedEx for the nextday environment as compared to the second-day environment. The highest drop height for UPS and USPS was higher for second-day shipments as compared to the next-day shipments. The average drop height of the ten highest drops experienced in the USPS shipping environment for Priority (second-day) service was higher than that for DHL, FedEx, and UPS.

As for the impact orientation for all shipments, for packages shipped next-day through all couriers, there were about 14-19 face impacts, about 1-2 impacts on the edge of the package, and up to 2 impacts on a corner for each one-way trip above $76 \mathrm{~mm}$. About $89 \%$ of all impacts were on a face, $5 \%$ were on 
an edge, and $5 \%$ were on a corner. Table 3 shows the information in more detail for all eight one-way trips. For packages shipped second-day through all couriers, there were about 8-20 face impacts, about 1-2 impacts on the edge of the package, and up to 2 impacts on a corner for each one-way trip above 76 $\mathrm{mm}$. About $88 \%$ of all impacts were on a face, $6 \%$ were on an edge, and $6 \%$ were on a corner. This contradicts earlier research [6] which found that the number of face, edge, and corner drops could be explained by elementary probabilities: since there are six faces, twelve edges, and eight corners in a typical box for a total of 26 possible impact orientations, the expected percentages for face, edge, and corner drops are $6 / 26$ or $23 \%, 12 / 26$ or $46 \%$, and $8 / 26$ or $31 \%$, respectively. The high incidence of face drops found in the present study suggests that these impacts are the result of automated handling operations, not drops.

\section{Recommended Test Procedures}

Singh and Burgess [10] have developed techniques in previous studies to use data from these types of measurement studies to develop lab simulation test methods. In most drop test methods there are three important parameters that control the outcome of the test results. These are:

- Drop height (severity) level

- Drop orientation

- Number of drops performed on the same test package

This paper provides data for the users to create test methods specific to the challenges of the distribution environment and the expected level of protection needed (or level of severity that packages get exposed to). Based on the previous studies drop height levels of $99 \%$ occurrence (Table 1) are generally used for either every expensive product or when extremely low levels of damage are desired. Other product types may select between 90 to $95 \%$ occurrence levels of drop heights based on either the value or allowable (acceptable) damage. In addition, the majority of drops in this study for this type of package size occurred on the face. It is therefore recommended to use the existing package face orientations to conduct drops as opposed to a larger percentage of edge and corner drops based on data in Table 3. The number of drops on the same package ranges from six, ten, and twelve based on current test methods of ASTM and International Safe Transit Association (ISTA). The data from Table 1 shows between 8 and 23 drops that each package observed among the various carriers and service measured above $76 \mathrm{~mm}$ ( 3 in.).

\section{Conclusions}

1. The number of drops experienced by the packages in the Overnight (next-day) environment for USPS was comparable with the average of those for DHL, FedEx, and UPS. The Priority (secondday) shipments in the USPS environment, however, experienced 2.3 times lesser drops as compared to the average of those experienced in DHL, FedEx, and UPS shipments.

2. While the highest drop height was greater for DHL and FedEx for the next-day environment as compared to the second-day environment, the highest drop height for UPS and USPS was higher for second-day (Priority) shipment as compared to the next-day (Express) shipment.

3. The average drop height of the ten highest drops experienced in the USPS shipping environment for both the Express (next-day) and Priority (second-day) service was higher than that for DHL, FedEx, and UPS.

4. The impact percentage for packages shipped next-day and second-day through all couriers was approximately the same for face, edge, and corner orientations. 


\section{References}

[1] www.USPS.com, 2006.

[2] www.DHL.com, 2006.

[3] www.fedex.com, 2006.

[4] www.ups.com, 2006.

[5] Goff, J., “Development of Performance Standards for Parcel Post Packages,” Michigan State University, Project No. $3108,1974$.

[6] Singh, S. P. and Voss, T., "Drop Heights Encountered in the United Parcel Service Small Parcel Environment in the United States,” J. Test. Eval., ASTM International,West Conshohocken, PA, Vol. 20, No. 5, 1992, pp. 382-387.

[7] Singh, S. P. and Cheema, A., "Measurement and Analysis of the Overnight Small Package Shipping Environment for Federal Express and United Parcel Service," J. Test. Eval.,ASTM International,West Conshohocken, PA, Vol. 24, No. 4, 1996, pp. $205-211$.

[8] Singh, P., Burgess, G., and Singh, J., "Measurement and Analysis of the Small and Light Weight Parcel Shipping Environment,” J. Test. Eval.,ASTM International,West Conshohocken, PA, Vol. 32, No. 5, 2004.

[9] Singh, P., Burgess, G., Singh, J., and Kremer, M., "Measurement and Analysis of the Next-day Air Shipping Environment for Midsized and Lightweight Packages for DHL, FedEx, and United Parcel Service," Journal of Packaging Technology and Science, Vol. 19, No. 4, 2006, pp. 227-235.

[10] Singh, S. P., Burgess, G., Hays, Z., "Measurement and Analysis of the UPS Ground Shipping Environment for Large and Heavy Packages," J. Test. Eval., ASTM International, West Conshohocken, PA, Vol. 29, 2001.

[11] Newsham, M. D., Pierce, S., and Singh, S. P., “Distribution, Parcel Labels Pose Challenges for Drop Orientation," Journal of Packaging Technology and Engineering, Vol. 8, No. 4, 1999, pp. 31-33.

[12] www.isthq.com, 2006. 


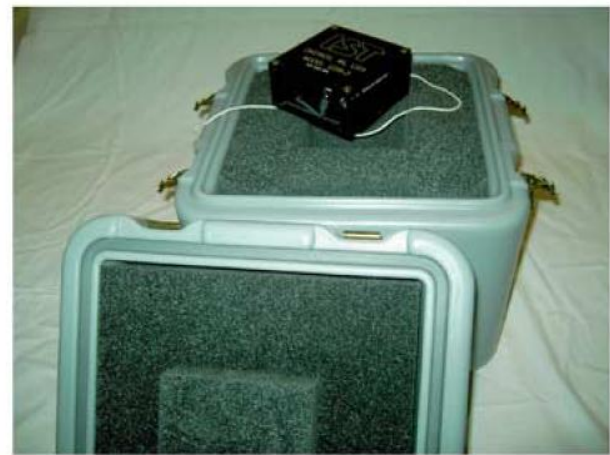

(a)

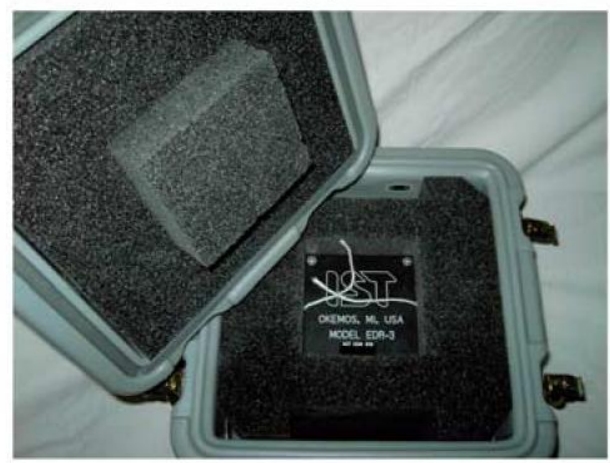

(b)

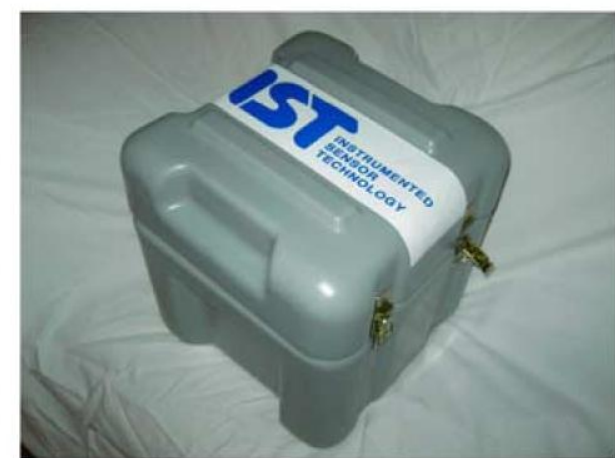

(c)

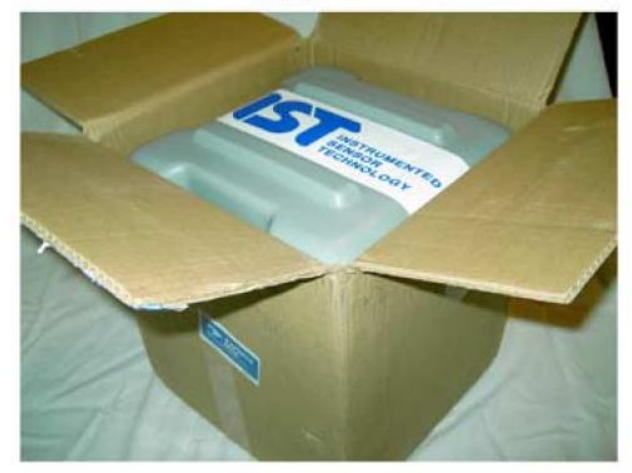

(d)

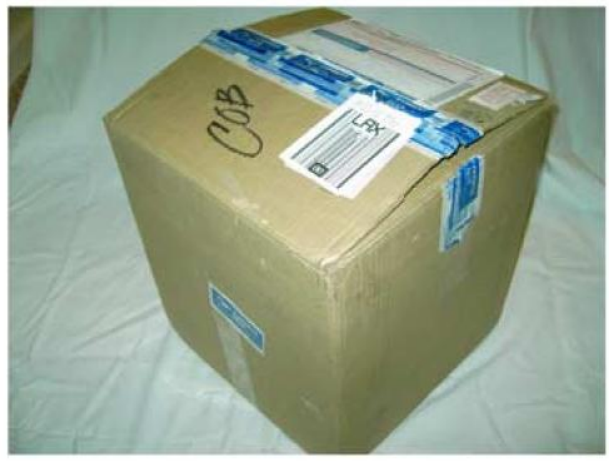

(e)

FIG. 1-Instrumented shipment preparation.

TABLE 1-Summary of drop height data measured above $76 \mathrm{~mm}$.

\begin{tabular}{|c|c|c|c|c|c|c|c|c|}
\hline \multirow[b]{2}{*}{ Drop Data } & \multicolumn{4}{|c|}{ Next-day or Express } & \multicolumn{4}{|c|}{ Second-day or Priority } \\
\hline & DHL & FedEx & UPS & USPS & DHL & FedEx & UPS & USPS \\
\hline Number of Drops & 184 & 128 & 117 & 156 & 168 & 182 & 104 & 66 \\
\hline Maximum Drop Height (m) & 1.45 & 1.77 & 1.23 & 1.43 & 1.01 & 0.89 & 1.63 & 1.87 \\
\hline Drop Height at $99 \%$ Occurrence (m) & 1.14 & 1.04 & 1.04 & 1.42 & 0.96 & 0.88 & 1.02 & 1.86 \\
\hline Drop Height at $95 \%$ Occurrence (m) & 0.86 & 0.7 & 0.68 & 0.76 & 0.76 & 0.66 & 0.76 & 1.16 \\
\hline Drop Height at $90 \%$ Occurrence (m) & 0.62 & 0.52 & 0.46 & 0.56 & 0.56 & 0.44 & 0.48 & 0.88 \\
\hline
\end{tabular}


TABLE 2-Drop height levels for shipments.

\begin{tabular}{|c|c|c|c|c|c|c|c|c|}
\hline \multirow[t]{2}{*}{ Drop Height (m) } & \multicolumn{4}{|c|}{ Next-day or Express } & \multicolumn{4}{|c|}{ Second-day or Priority } \\
\hline & DHL & FedEx & UPS & USPS & DHL & FedEx & UPS & USPS \\
\hline Highest & 1.45 & 1.77 & 1.23 & 1.43 & 1.01 & 0.89 & 1.63 & 1.87 \\
\hline $2^{\text {nd }}$ Highest & 1.14 & 1.03 & 1.02 & 1.43 & 0.98 & 0.89 & 1.00 & 1.65 \\
\hline $3^{\text {rd } H i g h e s t ~}$ & 1.04 & 1.00 & 0.92 & 1.00 & 0.90 & 0.86 & 0.94 & 1.34 \\
\hline $4^{\text {th }}$ Highest & 1.01 & 0.84 & 0.78 & 0.93 & 0.87 & 0.80 & 0.91 & 1.16 \\
\hline $5^{\text {th }}$ Highest & 0.95 & 0.76 & 0.72 & 0.90 & 0.86 & 0.69 & 0.82 & 1.14 \\
\hline $6^{\text {th }}$ Highest & 0.93 & 0.70 & 0.69 & 0.80 & 0.82 & 0.68 & 0.75 & 1.06 \\
\hline $7^{\text {th }}$ Highest & 0.93 & 0.69 & 0.62 & 0.77 & 0.81 & 0.68 & 0.62 & 0.88 \\
\hline $8^{\text {th }}$ Highest & 0.93 & 0.68 & 0.60 & 0.76 & 0.77 & 0.67 & 0.50 & 0.84 \\
\hline $9^{\text {th }}$ Highest & 0.85 & 0.62 & 0.59 & 0.74 & 0.75 & 0.66 & 0.48 & 0.76 \\
\hline $10^{\text {th }}$ Highest & 0.85 & 0.59 & 0.56 & 0.73 & 0.75 & 0.64 & 0.47 & 0.73 \\
\hline
\end{tabular}

TABLE 3-Percent orientation of impacts for packages for eight one-way trips.

\begin{tabular}{lcccr}
\hline & & \multicolumn{3}{c}{ Orientation of Drops (\%) } \\
\cline { 3 - 5 } \multicolumn{1}{c}{ Shipment Type } & & Face & Edge & Corner \\
\hline Next-day or Express & DHL & 83.69 & 7.61 & 8.69 \\
& FedEx & 91.41 & 6.25 & 2.34 \\
& UPS & 93.16 & 2.56 & 4.27 \\
& USPS & 92.95 & 3.21 & 3.85 \\
Second-day or Priority & DHL & 82.17 & 7.75 & 10.08 \\
& FedEx & 88.46 & 6.04 & 5.49 \\
& UPS & 93.27 & 4.81 & 1.92 \\
& USPS & 89.39 & 4.55 & 6.06 \\
\hline
\end{tabular}

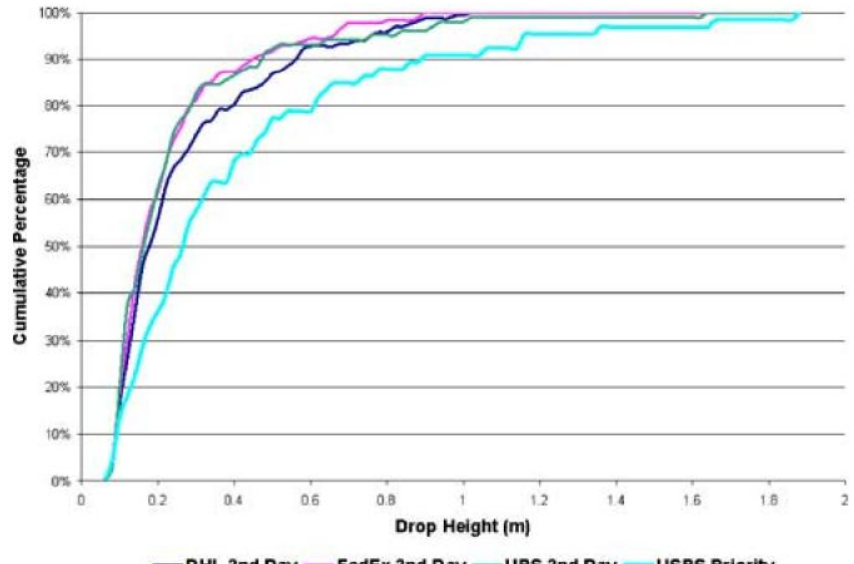

—DHL 2nd Day — FedEx 2nd Day — UPS 2nd Day — USPS Priority 


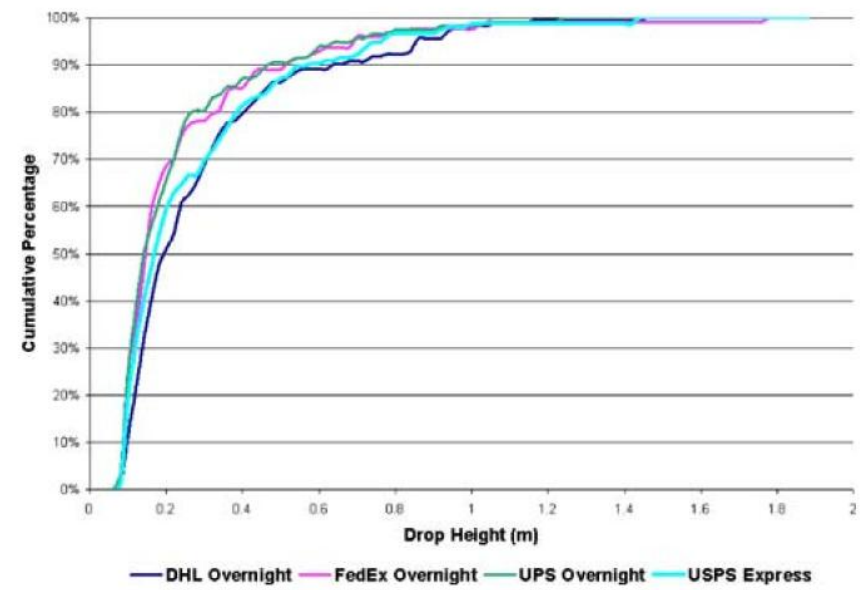

FIG. 2-Cumulative percentage versus drop height for next-day or express shipments. 